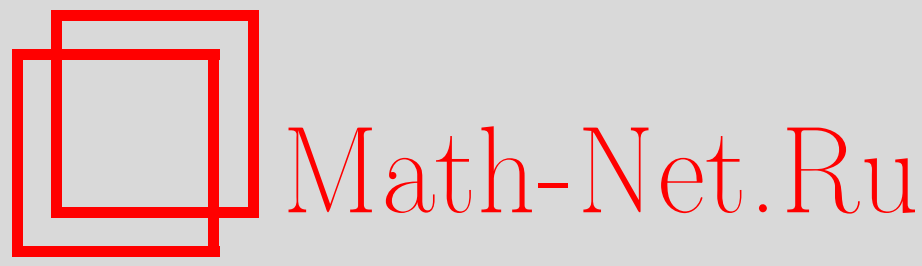

В. Г. Чирский, Представление натуральных чисел слагаемыми определенного вида, Совр. пробл. матем., 2017, выпуск 24, 81-84

DOI: https://doi.org/10.4213/spm70

Использование Общероссийского математического портала Math-Net.Ru подразумевает, что вы прочитали и согласны с пользовательским соглашением http://www . mathnet.ru/rus/agreement

Параметры загрузки:

IP: 54.164 .48 .24

26 апреля 2023 г., 11:44:52

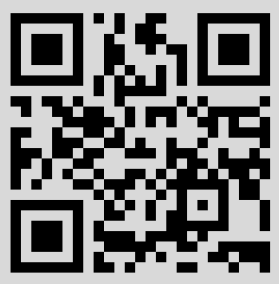




\title{
Представление натуральных чисел слагаемыми определенного вида
}

\author{
В.Г. Чирский \\ Московский государственный университет имени М.В.Ломоносова
}

Статья посвящена задаче о представлении натурального числа в виде суммы слагаемых определенного вида. Эта задача имеет давнюю историю. С ней связаны многие замечательные результаты и нерешенные проблемы.

Современные компьютерные технологии вызывают особый интерес к некоторым частным случаям этой задачи. Например, в алгоритмах возведения в степень часто используется представление натуральных чисел в системе с двумя основаниями (DBNS - double base number system). В этой задаче получены асимптотические оценки для количества членов в разложении натурального числа таким способом и для наименьшего натурального числа, не представимого заданным количеством слагаемых в системе с двумя основаниями.

В предлагаемой работе эти оценки значительно уточняются. Для этого используются очень точные оценки линейных форм от логарифмов, установленные Салиховым [1].

Введем необходимые обозначения. Пусть $P=\left\{p_{1}, \ldots, p_{t}\right\}$ обозначает множество, состоящее из конечного числа простых чисел $p_{1}, \ldots, p_{t}$. Пусть $A$ обозначает множество произведений целых неотрицательных степеней чисел $p_{1}, \ldots, p_{t}$. Множество $A_{ \pm}$определяется как $A \cup(-A)$.

Жарден и Наркевич [2] доказали, что не существует такого натурального числа $k$, что любое натуральное число $n$ можно представить суммой не более чем $k$ слагаемых из множества $A$.

Из теоремы Натансона [3] следует, что для любого натурального числа $k$ существует бесконечное множество целых чисел $n$ таких, что число $k$ представляет собой наименьшее значение $l$, для которых имеет место представление

$$
n=a_{1}+a_{2}+\cdots+a_{l}, \quad a_{1}, a_{2}, \ldots, a_{l} \in A_{ \pm} .
$$

Обозначим через $F(k)$-наименьшее натуральное число, которое нельзя представить в виде суммы менее чем $k$ слагаемых из множества $A$. Величина $F_{ \pm}(k)$ определяется аналогичным образом для множества $A_{ \pm}$. Исследуем задачу получения оценок для величин $F(k), F_{ \pm}(k)$. В работе [4] получены такие оценки для общего случая $P=\left\{p_{1}, \ldots, p_{t}\right\}$.

Tеорема 1 [4; теорема 2]. Пусть $P=\left\{p_{1}, \ldots, p_{t}\right\}$ - конечное множество простьх чисел и $A$ - множество произведений цельх неотрицательных степеней чисел из $P$. Пусть $k-$ натуральное число. Пусть $F(k)$ обозначает наименъшее натуральное число, которое нельзя представить в виде суммы $\sum_{i=1}^{k} a_{i} c a_{i} \in A \cup[0]$, а $F_{ \pm}(k)$ обозначает наименъшее натуральное число, которое нельзя представить в виде суммы $\sum_{i=1}^{k} a_{i}$ с $a_{i} \in A_{ \pm} \cup[0]$. Тогда для любого $\varepsilon>0$ существуют число с, зависящее только от двух наименьших чисел из $P$, число $C$, зависящее только от $\varepsilon, u$ абсолютная постоянная $C_{ \pm}$такие, что

1) $F(k)>k^{c k}$ для всех $k>1$;

2) $F(k) \leqslant C(k t)^{(1+\varepsilon) k t} \partial л я$ всех $k>1$;

3) $F_{ \pm}(k)<\exp \left((k t)^{C_{ \pm}}\right)$для всех $k>1$.

Для упомянутой выше задачи возведения в степень часто актуально представление натуральных чисел в системе с двумя основаниями: 2 и 3. 
Одна из важных проблем - получение асимптотической оценки длины представления числа $n$ суммой слагаемых вида $2^{a} 3^{b}$ с неотрицательными целыми $a$ и $b$. Для разложения используется «жадный» алгоритм, состоящий в том, что из числа $n$ вычитается наибольшее число вида $2^{a} 3^{b}$ с неотрицательными целыми $a$ и $b$. Затем с полученным числом производится та же процедура и т.д.

В работе [5] установлена следующая асимптотическая оценка.

ТЕОРема 2 [5]. Продолжительность работы приведенного выше алгоритма равна

$$
O\left(\frac{\log _{2} n}{\log _{2} \log _{2} n}\right), \quad n \rightarrow+\infty .
$$

В доказательстве этой теоремы существенно использована теорема Тайдемана [6], в которой основную роль играет оценка линейной формы от логарифмов чисел 2 и 3 . В работе [5] отмечено, что оценка для величины постоянной, подразумеваемой в этом асимптотическом соотношении, очень велика и далека от значений, получаемых при практических вычисленияx.

Именно за счет использования очень точных оценок линейных форм от логарифмов чисел 2 и 3, установленных Салиховым [1], и удается получить уточнение приведенных выше оценок.

Именно, в работе [7] доказано, что приведенное в теореме 2 асимптотическое соотношение выполняется при следующих условиях (формулируется несколько упрощенный и укороченный вариант теоремы из [7]).

ТЕОРема 3 [7]. При любом $C \geqslant 33 / 8$ и любом $\varepsilon>0$ при $n \geqslant N(C, \varepsilon)$ продолжителъность работы приведенного выше алгоритма не превосходит величины

$$
C_{0} \frac{\ln n}{\ln \ln n}
$$

где можно считать, что $C_{0}=6$.

Перейдем к уточнению оценок теоремы 1.

ТеОрема 4. Пусть А состоит из чисел вида $2^{a} 3^{b}$ с неотрицательными иельми $a$ u $b$. Множество $A_{ \pm}$определяется, как $A \cup(-A)$. Обозначим через $F(k)\left(F_{ \pm}(k)\right)$ - наименвшее натуральное число, которое нельзя представить в виде суммы менее чем $k$ слагаемьх из множества $A\left(A_{ \pm}\right)$. Тогда для любого $\varepsilon>0$ существует число $k_{0}$ такое, что при $k>k_{0}$ выполняются неравенства

$$
\left(\frac{k}{6}\right)^{k / 6} \leqslant F(k) \leqslant F_{ \pm}(k) \leqslant(2 k)^{(1+\varepsilon) 2 k} .
$$

ЗАмечАниЕ. Постоянная $k_{0}$ в этой теореме не является эффективной. Результат с эффективной постоянной $k_{0}$ имеет значительно худшую оценку сверху вида $F_{ \pm}(k)<\exp \left((2 k)^{C_{ \pm}}\right)$. Поскольку объектом исследования являлось получение асимптотически наиболее точной оценки, требование эффективности постоянной опущено. Отметим, что улучшение известного результата достигнуто в оценке снизу величины $F(k)$ за счет того, что была использована теорема Салихова [1] об оценке линейной формы от $\ln 2, \ln 3$. Оценка $F(k)>k^{c k}$, полученная на основе теоремы Тайдемана, имеет значительно меньшую, чем $1 / 6$, величину (порядка $0.000001)$.

Перейдем к доказательству теоремы 4 . Ввиду теоремы 3 количество слагаемых $k$ в представлении числа $n$ суммой слагаемых вида $2^{a} 3^{b}$ с неотрицательными целыми $a$ и $b$ удовлетворяет неравенству $k \geqslant 6 \ln n / \ln \ln n$, или $k \ln \ln n \geqslant 6 \ln n$, или $\ln (\ln n)^{k} \geqslant 6 \ln n$, откуда

$$
(\ln n)^{k} \leqslant n^{6} .
$$


Следовательно, используя неравенство $(1 / 6) k \ln \ln n \leqslant \ln n$, получаем

$$
n \geqslant(\ln n)^{k / 6} \geqslant\left(\frac{1}{6} k \ln \ln n\right)^{k / 6} \geqslant\left(\frac{1}{6} k\right)^{k / 6} .
$$

Таким образом, $F(k)$, т.е. наименьшее число, которое нельзя представить в виде суммы менее чем $k$ слагаемых из множества $A$, удовлетворяет неравенству

$$
F(k) \geqslant\left(\frac{1}{6} k\right)^{k / 6}
$$

Первая часть теоремы доказана.

Отметим очевидное неравенство $F(k) \leqslant F_{ \pm}(k)$ (если удалось представить число в виде суммы слагаемых из $A$, то мы получили представление и в виде суммы слагаемых из $\left.A_{ \pm}\right)$.

При получении оценки сверху для величины $F_{ \pm}(k)$ мы следуем доказательству теоремы 3 из работы [4]. В нем использована теорема Эвертса о подпространствах [8], которую мы сформулируем в следующем виде.

ЛЕмма [8; следствие 1]. Пусть числа $c, d$ удовлетворяют неравенствам $c>0,0 \leqslant d<1$. Пусть $S_{0}=\{2,3\}$, и пусть $l$ - натуральное число. Тогда существует лишь конечное число наборов челых чисел $\left(x_{0}, x_{1}, \ldots, x_{l}\right)$ таких, что НОД $\left(x_{0}, x_{1}, \ldots, x_{l}\right)=1$ и выполняется равенство

$$
x_{0}+x_{1}+\cdots+x_{l}=0
$$

а для любого собственного непустого подмножества $\left\{i_{0}, i_{1}, \ldots, i_{s}\right\}$ множества $\{0,1, \ldots, l\}$ имеет место неравенство

$$
x_{i_{0}}+x_{i_{1}}+\cdots+x_{i_{s}} \neq 0
$$

u, кроме того,

$$
\prod_{j=0}^{l}\left(\left|x_{j}\right|\left|x_{j}\right|_{2}\left|x_{j}\right|_{3}\right) \leqslant c\left(\max _{0 \leqslant j \leqslant l}\left|x_{j}\right|\right)^{d} .
$$

ЗАмечАниЕ. Символы $\left|x_{j}\right|_{2},\left|x_{j}\right|_{3}$ обозначают, соответственно, 2-адическое и 3-адическое нормирования целого числа $x_{j}$. Напомним, что если $p$ - простое число и целое число $x$ делится в точности на $p^{k}$, то $|x|_{p}=p^{-k}$.

Пусть $n$ - целое число, не делящееся ни на 2, ни на 3 , и пусть

$$
n=a_{1}+a_{2}+\cdots+a_{l}, \quad a_{1}, a_{2}, \ldots, \quad a_{l} \in A_{ \pm}, \quad l \leqslant k .
$$

Можно считать, что $l$ минимальное, так что любая сумма, состоящая не из всех слагаемых $a_{1}, a_{2}, \ldots, a_{l}$, не обращается в нуль. По определению множества $A_{ \pm} \operatorname{HOД}\left(a_{1}, a_{2}, \ldots, a_{l}\right)=1$. Применим лемму с $c=1, d=1 / 2$ к уравнению $a_{0}+a_{1}+\cdots+a_{l}=0$, где $a_{0}=-n$. Следовательно, для заданного $k$ существует лишь конечное число наборов целых чисел $\left(n, a_{1}\right.$, $\left.a_{2}, \ldots, a_{l}\right)$ с условиями, что $n$ - целое число, не делящееся ни на 2 , ни на 3, и $l \geqslant k$ таких, что

$$
n=a_{1}+a_{2}+\cdots+a_{l}, \quad a_{1}, a_{2}, \ldots, a_{l} \in A_{ \pm}, \quad n \leqslant\left(\max _{0 \leqslant j \leqslant l}\left|a_{j}\right|\right)^{1 / 2}, \quad n^{2} \leqslant \max _{0 \leqslant j \leqslant l}\left|a_{j}\right| .
$$

Пусть $N_{0}$ обозначает наибольшее из чисел $|n|$ для всех таких наборов (если таких наборов нет, то $\left.N_{0}=0\right)$. Рассмотрим натуральное число $n>N_{0}$, не делящееся ни на 2 , ни на 3 . Тогда для любого представления числа $n$ в виде

$$
n=a_{1}+a_{2}+\cdots+a_{l}, \quad a_{1}, a_{2}, \ldots, \quad a_{l} \in A_{ \pm}, \quad l \leqslant k,
$$


выполняются неравенства

$$
\left|a_{j}\right|<n^{2}, \quad j=1, \ldots, l .
$$

Представим $a_{j}= \pm 2^{s_{1}} 3^{s_{2}}$ и получим неравенство $\max \left\{s_{1}, s_{2}\right\}<2 \log _{2} n$. Количество таких возможных наборов $\left(a_{1}, a_{2}, \ldots, a_{l}\right)$ для заданного $l$ не превосходит числа $2^{l}\left(2 \log _{2} n\right)^{2 l}$, а возможных наборов $\left(a_{1}, a_{2}, \ldots, a_{l}\right), l \leqslant k$, не больше чем $2 \cdot 2^{k}\left(2 \log _{2} n\right)^{2 k}$. Тогда при $N>N_{0}$ существует не более чем $N_{0}+2 \cdot 2^{k}\left(2 \log _{2} N\right)^{2 k}$ натуральных чисел $n \leqslant N$, не делящееся ни на 2 , ни на 3 , имеющих представление в виде суммы не более чем $k$ слагаемых из $A_{ \pm}$. Количество натуральных чисел $n \leqslant N$, не делящееся ни на 2 , ни на 3 , не меньше, чем $N / 3$. Следовательно, для того, чтобы нашлось число $n \leqslant N$, не имеющее требуемого представления, достаточно, чтобы выполнялось неравенство

$$
\frac{N}{3}>N_{0}+2 \cdot 2^{k}\left(2 \log _{2} N\right)^{2 k}=N_{0} \cdot 2^{3 k+1}\left(\log _{2} N\right)^{2 k},
$$

или

$$
N>6 N_{0} \cdot 2^{3 k}\left(\log _{2} N\right)^{2 k}
$$

Следовательно,

$$
\log _{2} N>2 k\left(\log _{2} \log _{2} N+\frac{3}{2}+\log _{2} 6 N_{0}\right)
$$

или

$$
2 k<\frac{\log _{2} N}{\log _{2} \log _{2} N+C_{0}} .
$$

Пусть $N>(2 k)^{(1+\varepsilon) 2 k}$. Ввиду монотонности функции $\log _{2} x /\left(\log _{2} \log _{2} x+C_{0}\right)$ при больших $x$ при достаточно больших $k$ имеем

$$
\frac{\log _{2} N}{\log _{2} \log _{2} N+C_{0}}>\frac{(1+\varepsilon) 2 k \log _{2} 2 k}{\log _{2} 2 k+\log _{2}\left((1+\varepsilon) \log _{2} 2 k\right)+C_{0}}>2 k .
$$

Следовательно,

$$
F_{ \pm}(k) \leqslant(2 k)^{(1+\varepsilon) 2 k}
$$

Теорема доказана.

\section{Список литературы}

[1] В. Х. Салихов, “О мере иррациональности $\log 3$ ”, Докл. РАН, 417:6 (2007), 753-755.

[2] M. Jarden, W. Narkiewicz, "On sums of units", Monatsh. Math., 150:4 (2007), 327-332.

[3] M. B. Nathanson, "Geometric group theory and arithmetic diameter", Publ. Math. Debrecen, 79:3-4 (2011), 563-572.

[4] L. Hajdu, R. Tijdeman, "Representing integers as linear combinations of power products", Arch. Math. (Basel), 98:6 (2012), 527-533.

[5] V.S. Dimitrov, G. A. Julien, W. C. Miller, "An algorithm for modular exponentiation", Inform. Process. Lett., 66:3 (1998), 155-159.

[6] R. Tijdeman, "On the maximal distance between integers composed of small primes", Compositio Math., 28 (1974), 159-162.

[7] В. Г. Чирский, Р. Ф. Шакиров, "О представлении натуральных чисел с использованием нескольких оснований”, Чебышевский сб., 14:1 (2013), 86-93.

[8] J.-H. Evertse, "On sums of $S$-units and linear recurrences", Compositio Math., 53:2 (1984), 225-244. 